\title{
Meta-analysis on MTHFR polymorphism and lung cancer susceptibility in East Asian populations
}

\author{
XUE-DE ZHANG ${ }^{1}$, YAN-TING LI ${ }^{2}$, SHUAN-YING YANG ${ }^{1}$ and WEI LI ${ }^{1}$ \\ ${ }^{1}$ Department of Respiratory Medicine, The Second Affiliated Hospital, Xi'an Jiaotong University, Xi'an, Shannxi 710004; \\ ${ }^{2}$ Department of Ultrasonography, Tongchuan City People's Hospital, Tongchuan, Shannxi 727000, P.R. China
}

Received December 19, 2012; Accepted February 11, 2013

DOI: $10.3892 /$ br. 2013.68

\begin{abstract}
Lung cancer is the most frequently occurring type of cancer worldwide and the leading cause of cancer mortality. Environmental and genetic factors play important roles in lung carcinogenesis. The aim of this meta-analysis was to investigate the association between methylenetetrahydrofolate reductase (MTHFR) polymorphism and the risk of lung cancer in East Asian populations. Related articles were identified through searching literature databases, such as PubMed, EMBASE, Web of Science, Chinese Biomedicine and CNKI. The odds ratio (OR) values in those studies were incorporated by meta-analysis to assess lung cancer susceptibility associated with the MTHFR mutation genotype. The MTHFR C677TT genotype exhibited a significantly increased risk of lung cancer compared to the MTHFR 677CC/CT genotype $(\mathrm{OR}=1.24 ; 95 \% \mathrm{CI}, 1.01-1.52)$. No relationship was identified between the other MTHFR C677T genetic models and the risk of lung cancer and there was no significantly increased risk of lung cancer in A1298C genetic models. In a subgroup of hospital-based controls, according to the source of controls, the C677TT genotype exhibited a significantly increased risk of lung cancer, compared to the $\mathrm{C} 677 \mathrm{CC}$ genotype $(\mathrm{OR}=3.01$; $95 \% \mathrm{CI}, 1.07-8.46)$. In the stratified analysis, the study indicated that the MTHFR 677TT genotype was associated with a significant increase in the risk of lung squamous carcinoma (OR=1.53; 95\% CI, 1.09-2.14), whereas no association was observed between the MTHFR C677TT genotype and the risk of lung adenocarcinoma. No association was observed between MTHFR C677TT polymorphism and the risk of lung cancer when smoking was considered. In conclusion, the meta-analysis results suggested that MTHFR C677T polymorphisms exhibit a significantly increased risk of lung cancer and that
\end{abstract}

Correspondence to: Professor Shuan-Ying Yang, Department of Respiratory Medicine, the Second Affiliated Hospital of Medical College, Xi'an Jiaotong University, 157 Xi 5 Road, Xi'an, Shannxi 710004, P.R. China

E-mail: yangshuanying66@163.com

Key words: lung cancer, methylenetetrahydrofolate reductase, polymorphisms, meta-analysis the MTHFR 677TT genotype is associated with a significantly increased risk of lung squamous carcinoma.

\section{Introduction}

Lung cancer is the most frequently occurring type of cancer worldwide and the leading cause of cancer mortality, with a five-year survival rate $\leq 15 \%$ (1). Smoking is the primary risk factor for lung cancer. However, lung cancer develops in $<20 \%$ of smokers (2). This suggests that environmental and genetic factors play important roles in lung carcinogenesis. Over the past few decades, the relationship between environmental and genetic factors and cancer susceptibility has been widely investigated. Folate metabolism is considered to be important role in carcinogenesis through its involvement in the process of DNA methylation and repair. Methylenetetrahydrofolate reductase (MTHFR) is located in the short arm of chromosome 1 (1p36.3) and is involved in DNA methylation and synthesis as an important enzyme in the folic acid metabolic process. C677T (exon 4) and A1298C (exon 7) are two common polymorphisms in the MTHFR gene, which decrease enzyme activity compared to the wild-type gene, leading to DNA repair and methylation disorders and playing an important role in carcinogenesis (3). Numerous epidemiological studies investigated the association between MTHFR C677T and A1298C gene polymorphism and lung cancer predisposition. However, the results were inconsistent, possibly due to the small sample size, selection bias or other factors. Meta-analysis is a valid method to assess disparate results. In 2008, Mao et al (4) performed a meta-analysis that included eight articles; however, they did not identify a significant correlation between the MTHFR polymorphisms and lung cancer globally. The ethnic lines involved in the meta-analysis were diverse, including Asians, non-Hispanic whites and Europeans. MTHFR mutation frequency is diverse between various ethnicities. The incidence of the C677T homozygous mutant is $20 \%$ in Chinese, $18 \%$ in Italian and $11 \%$ in African populations (5). Lung cancer morbidity, histological type and molecular typing also differ among different ethnic lines. To the best of our knowledge, there is currently no meta-analysis available on MTHFR and lung cancer susceptibility in East Asian populations. Over the past few years, newly published studies were conducted, most of which involved Japanese, South Korean and Chinese populations. In order to reduce research bias, we performed a meta-analysis with the studies 
involving East Asian populations of identical race, to investigate the relationship between MTHFR gene polymorphism and lung cancer susceptibility.

\section{Materials and methods}

Publication search. To identify all the studies investigating the association between MTHFR gene polymorphism and lung cancer susceptibility, computer searches of literature databases, such as PubMed, EMBASE, Web of Science, Chinese Biomedicine and CNKI (prior to November 30, 2012) were performed, using the following $\mathrm{MeSH}$ terms and key words: 'methylenetetrahydrofolate reductase' or 'MTHFR' and 'lung' and 'cancer' or 'carcinoma'. The studies were read in their entirety and their references were also reviewed to identify other relevant studies.

Inclusion and exclusion criteria. Eligible studies were required to meet the following criteria: i) exploration of MTHFR polymorphisms and lung cancer risk in East Asian population; ii) unrelated case-control or cohort studies; iii) sufficient published data for estimating an odds ratio (OR) with $95 \%$ confidence intervals (CIs); iv) allelic distributions in the control group consistent with the Hardy-Weinberg equilibrium (HWE). Exclusion criteria were as follows: i) studies that did not provide the population sources of cases and controls; ii) when more than one study involved the same sample population, only the best quality one was included.

Data extraction. Information was carefully extracted from the eligible publications according to the inclusion criteria listed above. The following data were collected from each study: first author's surname, year of publication, country, sample size, source of controls, genotyping method, number of cases and controls for each C677T or A1298C genotype, smoking status, histological type and alcohol consumption.

Statistical analysis. HWE was tested by the Chi-square test. $\mathrm{P}<0.05$ was considered to indicate a statistically significant difference (6). Crude ORs with their corresponding 95\% CIs were used to assess the association between MTHFR C677T and A1298C polymorphisms and lung cancer risk (7). The pooled ORs were estimated for MTHFR C677T genotypes and MTHFR A1298C genotypes. The heterogeneity between studies was assessed using the Q statistic (8). When heterogeneity was considered to be significant $(\mathrm{P}<0.10)$, the random-effects model was used. In the case of non-significant heterogeneity $(\mathrm{P} \geq 0.10)$, the fixed-effects model was used. We quantified heterogeneity using the $\mathrm{I}^{2}$ metric score $\left(\mathrm{I}^{2}<25 \%\right.$, no heterogeneity; $\mathrm{I}^{2}=25-50 \%$, moderate heterogeneity; and $\mathrm{I}^{2}>50 \%$, significant heterogeneity) (9). Subgroup analyses were also performed on the basis of the source of controls, smoking status and histological types. Sensitivity analysis was conducted to determine whether the exclusion of any one study affected the initial results. Funnel plots were used to assess publication bias. An asymmetric plot suggested possible publication bias. Egger's linear regression test (10) was performed to determine the significance of the asymmetry. $\mathrm{P}<0.05$ was considered to indicate publication bias. Analyses were performed using Review Manager software version 5.0.

\section{Results}

Study characteristics. A total of 72 studies were preliminarily retrieved based on the search terms and 13 studies (11-23) met the inclusion criteria for detailed analysis following abstract scanning and full-text assessment (Fig. 1). The possible eligible papers were retrieved by two independent investigators (X.D. Zhang and W. Li). All eligible articles provided data on C677T and seven of them also provided data on A1298C. The main characteristics of the studies are listed in Table I. However, A1298C allelic distributions in the control population of one study (21) significantly deviated from HWE. Thirteen studies (7,409 cases and 6,331 controls) on C677T genotypes, among which there were six articles (2,049 cases and 2,851 controls) on A1298C genotypes, were included in our meta-analysis. The included studies had all been conducted on East Asian populations. Controls of four studies $(11,12,18,19)$ were derived from hospital-based participants and controls of nine studies (13-17,20-23) were derived from population-based participants.

Quantitative synthesis. There was significant heterogeneity $\left(\mathrm{P}<0.0001, \mathrm{I}^{2}=71 \%\right)$ among the 13 studies on C677T. To eliminate heterogeneity, we subgrouped the 13 studies based on the sources of controls; however, the heterogeneity was not eliminated. The pooled ORs were estimated using the random-effects model. The results demonstrated a significantly increased risk of lung cancer in the MTHFR 677TT compared to the C677CC/CT genotype $(\mathrm{OR}=1.24 ; 95 \% \mathrm{CI}, 1.01-1.52)$ (Fig. 2). No statistical significance was observed between other C677T gene models and lung cancer (Table II). In a subgroup of hospital-based controls, the C677TT genotype exhibited a significantly increased risk of lung cancer compared to the C677CC genotype (OR=3.01; 95\% CI, 1.07-8.46). There was no significant association for population-based controls $(\mathrm{OR}=1.02$; 95\% CI, 0.55-1.89). Two published studies on MTHFR polymorphism and lung cancer risk in smokers provided detailed data. In the stratified analysis, no association was found between MTHFR C677TT polymorphism, compared to $\mathrm{C} 677 \mathrm{CC} / \mathrm{CT}$, and the risk of lung cancer, regardless of the smoking status. However, the study indicated that the MTHFR $677 \mathrm{TT}$ genotype is associated with a significant increase in the risk of lung squamous carcinoma, compared to $\mathrm{C} 677 \mathrm{CC} / \mathrm{CT}$ $(\mathrm{OR}=1.53 ; 95 \% \mathrm{CI}, 1.09-2.14)$, whereas no association was found between the MTHFR C677TT genotype and the risk of lung adenocarcinoma (Table III). Stratified analysis based on alcohol consumption and folate intake was not performed, since no sufficient data were reported. We performed a sensitivity analysis by sequentially omitting individual studies. As regards the C677T genotype, there was no significant deviation from the initial ORs and no significant association between C677T polymorphism and the risk of lung cancer was observed. As regards the A1298C polymorphism, heterogeneity was not observed among the six studies. There was no significant relationship between A1298C polymorphism and lung cancer risk by the fixed-effects model (Table II). There was also no significant association in the subgroup analysis according to the control source. We did not perform a stratified analysis based on smoking status, histological type, alcohol consumption and folate intake, since no sufficient data were provided by A1298C studies. The relationships between the 


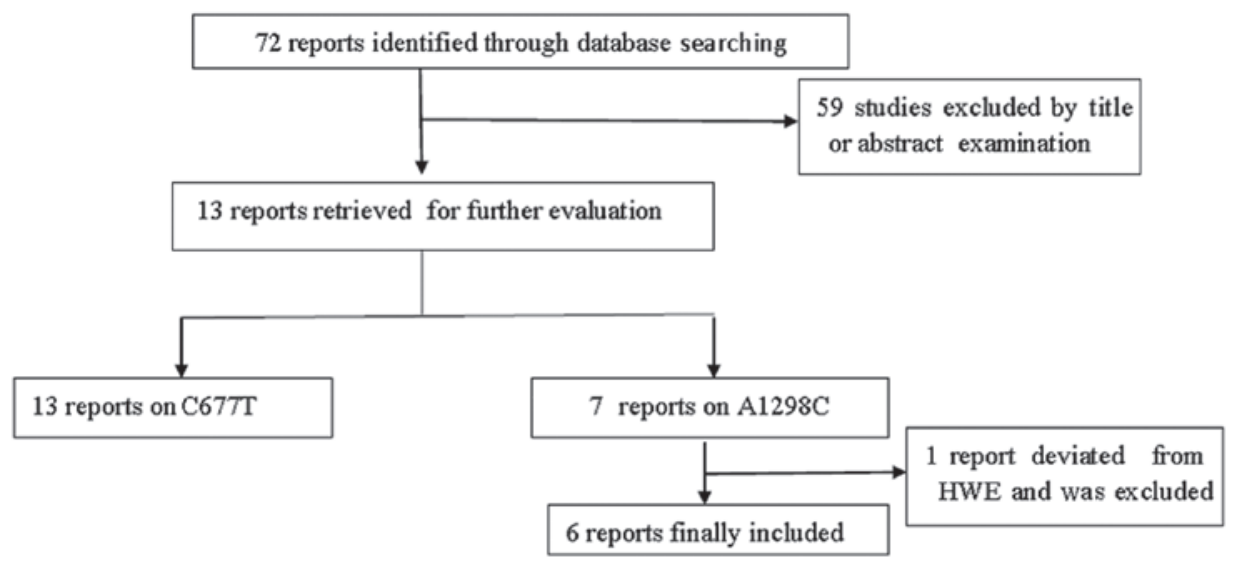

Figure 1. Study flow chart for the process of selecting the eligible publications.

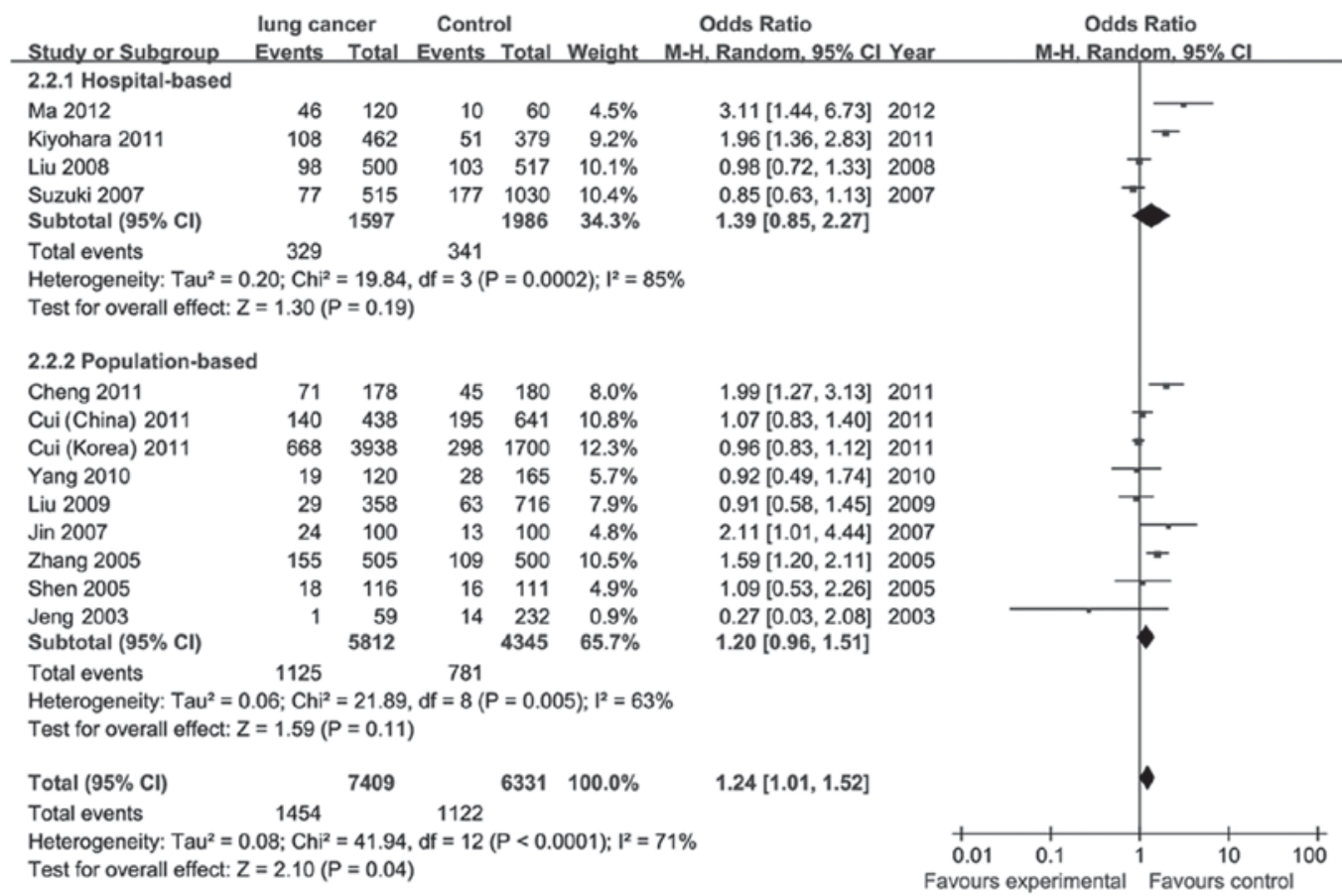

Figure 2. Forest plot of studies on the association between methylenetetrahydrofolate reductase (MTHFR) C677T polymorphisms (TT vs. CC/CT) and lung cancer.

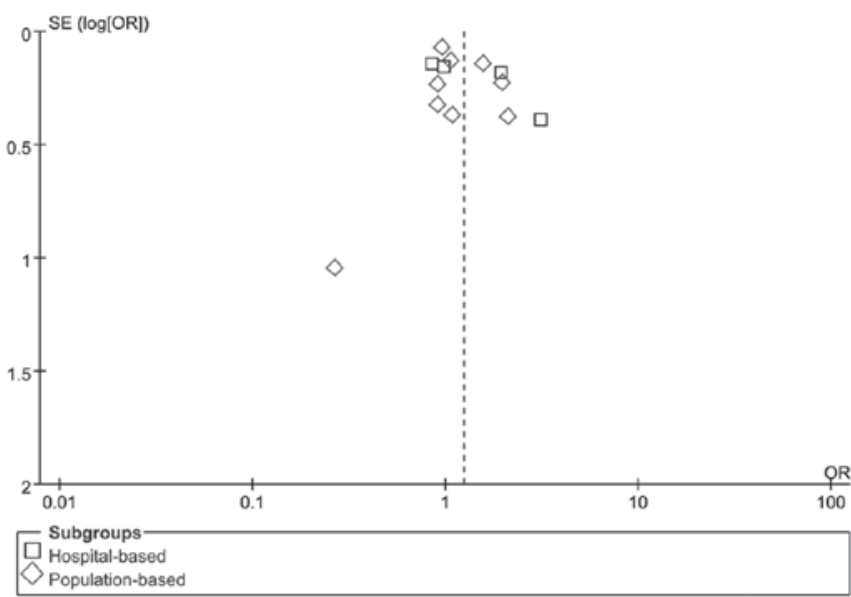

Figure 3. Funnel plot of studies on the association between methylenetetrahydrofolate reductase (MTHFR) C677T polymorphisms (TT vs. CC/CT) and lung cancer.
A1298C polymorphisms and the risk of lung cancer were not altered during the sensitivity analysis.

Publication bias. RevMan funnel plots and Egger's tests were performed to assess publication bias. The shapes of the funnel plots did not reveal any obvious sign of asymmetry for C677TT vs. CC/CT (Fig. 3). Furthermore, Egger's test was used to provide statistical evidence of funnel plot symmetry. The results revealed no evidence of publication bias $(\mathrm{P}=0.33)$. No evidence of publication bias was observed in other gene models. The results suggested that the data provided by these meta-analyses are relatively reliable.

\section{Discussion}

Epidemiological studies have demonstrated that environmental and genetic factors may contribute to the development 


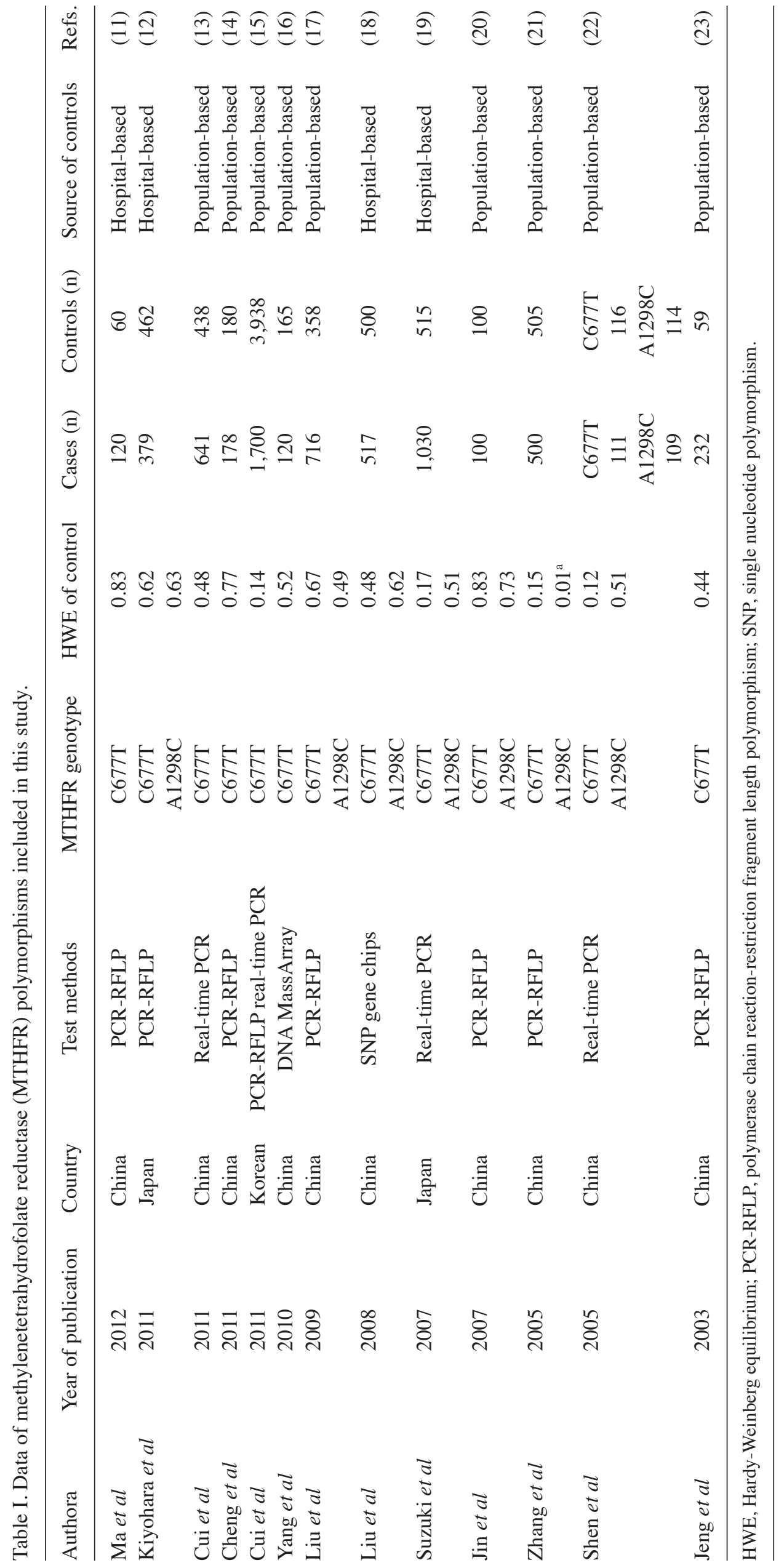


Table II. Meta-analysis of methylenetetrahydrofolate reductase (MTHFR) polymorphisms and risk of lung cancer.

\begin{tabular}{|c|c|c|c|}
\hline Genetic models & OR $(95 \% \mathrm{CI})$ & $\mathrm{I}^{2}(\%)$ & P-value \\
\hline \multicolumn{4}{|l|}{ C677T } \\
\hline \multicolumn{4}{|l|}{ TT vs. CC/CT } \\
\hline All & $1.24(1.01-1.52)$ & 71 & 0.0001 \\
\hline Hospital-based & $1.39(0.85-2.27)$ & 85 & 0.0002 \\
\hline Population-based & $1.20(0.90-1.51)$ & 63 & 0.005 \\
\hline \multicolumn{4}{|l|}{ TT vs. CC } \\
\hline All & $1.44(0.86-2.39)$ & 94 & 0.00001 \\
\hline Hospital-based & $3.01(1.07-8.46)$ & 94 & 0.00001 \\
\hline Population-based & $1.02(0.55-1.89)$ & 94 & 0.00001 \\
\hline \multicolumn{4}{|l|}{ TT/CT vs. CC } \\
\hline All & $1.15(0.84-1.56)$ & 92 & 0.00001 \\
\hline Hospital-based & $1.26(0.90-1.74)$ & 77 & 0.004 \\
\hline Population-based & $1.08(0.71-1.63)$ & 93 & 0.00001 \\
\hline \multicolumn{4}{|l|}{ A1298C } \\
\hline \multicolumn{4}{|l|}{ CC vs. AA/AC } \\
\hline All & $1.18(0.88-1.59)$ & 0 & 0.48 \\
\hline Hospital-based & $1.24(0.88-1.76)$ & 0 & 0.44 \\
\hline Population-based & $1.02(0.57-1.82)$ & 25 & 0.26 \\
\hline \multicolumn{4}{|l|}{ CC vs. AA } \\
\hline All & $1.18(0.88-1.59)$ & 0 & 0.45 \\
\hline Hospital-based & $1.24(0.87-1.75)$ & 4 & 0.35 \\
\hline Population-based & $1.04(0.58-1.86)$ & 20 & 0.29 \\
\hline \multicolumn{4}{|l|}{ CC/AC vs. AA } \\
\hline All & $1.02(0.90-1.15)$ & 0 & 0.76 \\
\hline Hospital-based & $1.01(0.87-1.17)$ & 22 & 0.28 \\
\hline Population-based & $1.04(0.90-1.15)$ & 0 & 0.76 \\
\hline
\end{tabular}

OR, odds ratio; CI, confidence interval.

Table III. Stratified analyses based on smoking and histology type of the association between methylenetetrahydrofolate reductase (MTHFR) C677T polymorphisms (TT vs. CC/CT) and lung cancer.

\begin{tabular}{|c|c|c|c|c|c|c|c|}
\hline \multirow{2}{*}{$\begin{array}{l}\text { Subgroup } \\
\text { analyses }\end{array}$} & \multicolumn{2}{|c|}{ Cases } & \multicolumn{2}{|c|}{ Controls } & \multirow[b]{2}{*}{ OR (95\% CIs) } & \multirow[b]{2}{*}{ P-value } & \multirow[b]{2}{*}{ Refs. } \\
\hline & $\mathrm{TT}$ & $\mathrm{CC} / \mathrm{CT}$ & $\mathrm{TT}$ & $\mathrm{CC} / \mathrm{CT}$ & & & \\
\hline Ever smoker & 104 & 498 & 72 & 661 & $1.38(0.59-3.21)$ & 0.46 & $(12,17)$ \\
\hline Never smoker & 55 & 246 & 42 & 220 & $1.28(0.78-2.10)$ & 0.33 & $(12,17)$ \\
\hline Squamous carcinoma & 103 & 131 & 137 & 392 & $1.53(1.09-2.14)$ & 0.01 & $(11,13,14)$ \\
\hline Adenocarcinoma & 88 & 142 & 137 & 392 & $2.13(0.90-5.00)$ & 0.08 & $(11,13,14)$ \\
\hline
\end{tabular}

OR, odds ratio; CI, confidence interval.

of the vast majority of tumors. Previously published studies demonstrated that MTHFR polymorphisms were associated with breast (24), colon (25), gastric (26) and other types of cancer. MTHFR may play an important role in folate metabolism as a key enzyme, by irreversibly catalyzing the generation of 5,10-methylenetetrahydrofolate $\left(5,10-\mathrm{CH}_{2}-\mathrm{THF}\right)$ to 5-methylenetetrahydrofolate $\left(5-\mathrm{CH}_{3}-\mathrm{THF}\right)$, which is a methyl group donor for DNA repair and synthesis. The lack of a methyl group leads to genome-wide hypomethylation, which affects the stability of the genome and may easily lead to malignant transformation of normal cells (27). Genome-wide hypomethylation is an important characteristic of cancer cells. 
$5,10-\mathrm{CH}_{2}$-THF is a substrate of the thymidylate synthetase enzyme, is involved in purine synthesis in the methylation of deoxyuridine monophosphate (dUMP) nucleotide to deoxythymidine monophosphate (dTMP) and contributes to DNA synthesis. In the case of dTMP shortage, it is replaced by dUMP in DNA synthesis, leading to DNA double-strand breaks and resulting in tumorigenesis (28). MTHFR C677T and A1298C are two common genetic polymorphisms. The MTHFR variant genotype C677TT decreases enzymatic activity by $70 \%$ (3) and C677CT by 40\% (29). MTHFR A1298C polymorphism also reduces the specific activity of the enzyme, although to a lesser extent. Decreased enzymatic activity of MTHFR affects folate metabolism. Folate facilitates the provision of methyl groups that are required for intracellular methylation reactions and DNA repair. Thus, MTHFR polymorphism may increase the risk of lung cancer, as well as of other malignancies (30). Published data from recent studies demonstrated a significant correlation between MTHFR gene polymorphism and the risk of lung cancer (11-14,17,20-23,31-33). However, other studies failed to demonstrate such a correlation $(15,16,18,19,34-36)$. The meta-analysis may provide comprehensive evaluation and quantitive analysis of inconsistent results from individual studies of identical aim. We conducted the meta-analysis to elucidate the association of MTHFR C677T and A1298C polymorphisms with susceptibility to lung cancer in East Asian populations. The results indicate a significantly increased risk of lung cancer associated with the MTHFR 677TT genotype compared to the MTHFR $677 \mathrm{CC} / \mathrm{CT}$ genotypes, but indicated no positive relationship between other MTHFR C677T genetic models or the A1298 genetic model and the risk of lung cancer, when the studies were pooled together. The harmful substances in tobacco may cause DNA damage, leading to gene mutations, thus increasing the risk of lung and esophageal cancer, as well as of other malignancies; by contrast, the adequate intake of folate significantly reduces the risk of lung cancer (22). Therefore, folate metabolism and smoking are crucial in the occurrence of lung cancer. In the stratified meta-analysis based on smoking status, we did not observe a positive relationship between MTHFR C677T gene polymorphism and lung cancer risk, regardless of the smoking status. The results suggested that smoking was not a predominant factor affecting the association between MTHFR C677T polymorphisms and lung cancer risk. Of note, only two published studies provided sufficient data to assess the relationship between MTHFR polymorphism and lung cancer risk in the presence of smoking. Therefore, the results are not considered reliable. In the stratified analysis based on histological types, we observed that the MTHFR 677TT genotype is associated with a significant increase in the risk of lung squamous carcinoma compared to $\mathrm{C} 677 \mathrm{CC} / \mathrm{CT}$, but is negative in lung adenocarcinoma. The results suggest that MTHFR C677T polymorphisms may be related to histological type. We were not able to perform a stratified analysis based on alcohol consumption and folate intake, due to the insufficient data.

Heterogeneity may affect the interpretation of the meta-analysis results. There was significant heterogeneity for C677T among the 13 studies. Heterogeneity is generally divided into clinical, methodological and statistical heterogeneity (37), which may lead to a meta-analysis heterogeneity. Clinical heterogeneity includes differences in the studied populations, sample selection (e.g., source of control populations) and other risk factors. Methodological heterogeneity may be mostly due to differences in trial design and quality. Statistical heterogeneity is due to the variation of the treatment effects estimated among different trials. Among these, clinical heterogeneity is the most common reason for meta-analysis heterogeneity. The identification of the source of heterogeneity is one of the most important aims of a meta-analysis. Therefore, we selected East Asian populations of identical race, leading similar lifestyles and exposed to similar environmental factors, in order to perform this meta-analysis. We also stratified the studies according to the source of controls. Subsequently, a subgroup analysis stratified according to the source of control population demonstrated a significant heterogeneity as well, suggesting that the source of the control population contributed only slightly to the overall heterogeneity. In the subgroup analysis based on the source of the control population, we observed a significantly increased risk of lung cancer associated with the C677TT genotype, compared to the C677CC genotype, in a subgroup of hospital-based controls. However, we were unable to reach definitive conclusions due to the small sample size of this study and the complexity of the individuals included in the control groups, who may suffer from other diseases associated with MTHFR mutations. Therefore, more detailed information should be obtained to evaluate the relationship among them. Heterogeneity may be due to the source of control population including, not only hospital patients, but the general population as well; subjects in the general population may suffer from other diseases associated with MTHFR gene polymorphism, such as hypertension (38), cardiovascular and cerebrovascular diseases (39).

Certain limitations of this meta-analysis should be acknowledged. Firstly, some individuals in the control group are likely to suffer from diseases associated with MTHFR gene polymorphism. Secondly, some individuals in the control group may develop lung cancer years later, although they were negative for signs of lung cancer at the time of the investigation. Thirdly, we were not able to conduct a subgroup analysis according to age, folate intake and alcohol consumption, due to the sparsity of available data on lung cancer subtype. Finally, subgroup analyses according to smoking status and histological type were performed, but the sample size was insufficient to support the results. Despite these limitations, the meta-analysis included a substantial number of cases and controls from the studies on the association between MTHFR polymorphism and lung cancer risk, which greatly enhanced the statistical power of the analysis and provided enough evidence for the authors to draw a reliable conclusion. Furthermore, no publication bias was identified in the present meta-analysis, which enhances the reliability of the meta-analysis results.

In conclusion, this meta-analysis indicated that MTHFR $677 \mathrm{~T}$ polymorphism has a significant association with lung cancer risk. However, since the number of the studies included in our meta-analysis was limited, large-sample studies using standardized unbiased methods, definitively diagnosed lung cancer patients, more detailed individual data and well-matched controls should be conducted. Moreover, lung cancer development results from the interaction of genetic and environmental factors. Future studies should consider 
gene-gene and gene-environment interactions in lung cancer Further investigations on MTHFR polymorphisms and lung cancer susceptibility may elucidate the relationship between the two and provide a theoretical basis for lung cancer prevention.

\section{Acknowledgements}

This study was supported by the National Natural Science Foundation of China (no. 81172234).

\section{References}

1. Jemal A, Siegel R, Ward E, Hao Y, Xu J, and Thun M: Cancer statistics. CA Cancer J Clin 59: 225-249, 2009.

2. Schwartz AG: Lung cancer: family history matters. Chest 130: 936-937, 2006

3. van der Put NM, Gabreels F, Stevens EM, et al: A second common mutation in the methylenetetrahydrofolate reductase gene: an additional risk factor for neural-tube defects? Am J Hum Genet 62: 1044-1051, 1998.

4. Mao RF, Fan Y, Jin Y, Bai J and Fu S: Methylenetetrahydrofolate reductase gene polymorphisms and lung cancer: a meta-analysis. J Hum Genet 53: 340-348, 2008.

5. Curtin K, Bigler J, Slattery ML, Caan B, Potter JD and Ulrich CM: MTHFR C677T and A1298C polymorphisms: diet, estrogen, and risk of colon cancer. Cancer Epidemiol Biomarkers Prev 13: 281-292, 2004

6. Guo SW and Thompson EA: Performing the exact test of Hardy-Weinberg proportion for multiple alleles. Biometrics 48 361-372, 1992

7. Woolf B: On estimating the relation between blood group and disease. Ann Hum Genet 19: 251-253, 1995.

8. Zintzaras E and Ioannidis JP: Heterogeneity testing in meta-analysis of genome searches. Genet Epidemiol 28: 123-137, 2005.

9. Higgins JP and Thompson SG: Quantifying heterogeneity in a meta-analysis. Stat Med 21: 1539-1558, 2002

10. Egger M,Davey Smith G,Schneider Mand Minder C: Biasin meta-analysis detected by a simple, graphical test. BMJ 315 : 629-634, 1997.

11. Ma QL, Li YF, Ji M, Yang KY, Wang JY, Li S and Huang YC: The relationship of Methylenetetrahydrofolate reductase SNP677C_T and lung cancer susceptibility. Chin J Clinicians (Electronic Edition) 6: 213-215, 2012 (In Chinese).

12. Kiyohara C, Horiuchi T, Takayama K and Nakanishi $\mathrm{Y}$ Methylenetetrahydrofolate reductase polymorphisms and interaction with smoking and alcohol consumption in lung cancer risk: a case-control study in a Japanese population. BMC Cancer 11: 459, 2011.

13. Cui LH, Yu Z, Zhang TT, Shin MH, Kim HN and Choi JS: Influence of polymorphisms in MTHFR $677 \mathrm{C} \rightarrow \mathrm{T}$, TYMS $3 \mathrm{R} \rightarrow 2 \mathrm{R}$ and MTR $2756 \mathrm{~A} \rightarrow \mathrm{G}$ on NSCLC risk and response to platinum-based chemotherapy in advanced NSCLC. Pharmacogenomics 12: 797-808, 2011.

14. Cheng Z, Wang W, Song YN, et al: Investigation of the relationship between C677T polymorphisms of methylenetetrahydrofolate reductase and lung cancer risk. Chin J Tuberc Respir Dis 34: 57-58, 2011 (In Chinese).

15. Cui LH, Shin MH, Kim HN, et al: Methylenetetrahydrofolate reductase $\mathrm{C677T}$ polymorphism in patients with lung cancer in a Korean population. BMC Med Genet 12: 28, 2011

16. Yang XX, Li FX, Yi J, Li X, Sun JZ and Hu NY: An association of methylenetetrahydrofolate reductase reductase C677T and gastric cancer, colorectal cancer and lung cancer susceptibility. Guangdong Med J 31: 2375-2377, 2010 (In Chinese)

17. Liu CS, Tsai CW, Hsia TC, et al: Interaction of methylentetrahydrofolate reductase genotype and smoking habit in Taiwanese lung cancer patients. Cancer Genomics Proteomics 6: 325-329, 2009.

18. Liu H, Jin G, Wang $\mathrm{H}$, et al: Association of polymorphisms in one-carbon metabolizing genes and lung cancer risk: a case-control study in Chinese population. Lung Cancer 61: 21-29, 2008.

19. Suzuki T, Matsuo K, Hiraki A, et al: Impact of one-carbon metabolism-related gene polymorphisms on risk of lung cancer in Japan: a casecontrol study. Carcinogenesis 28: 1718-1725, 2007.
20. Jin C, Zhang YH, Peng MF, Li WD, Ma L and Chen WS: Study of the relationship between C677T, A1298C gene polymorphisms of methylenetetrahydrofolate reductase and lung cancer. Chin Clin Oncol 12: 671-675, 2007.

21. Zhang XM, Miao XP, Tan W, Qu SN, Sun T, Zhou YF and Lin DX: Association between genetic polymorphisms in methylentetrahydrofolate reductase and risk of lung cancer. Acta Academiae Medicinae Sinicae 27: 700-703, 2005 (In Chinese)

22. Shen M, Rothman N, Berndt SI, et al: Polymorphisms in folate metabolic genes and lung cancer risk in Xuan Wei, China. Lung Cancer 49: 299-309, 2005.

23. Jeng YL, Wu MH, Huang HB, et al: The methylenetetrahydrofolate reductase $677 \mathrm{C} \rightarrow \mathrm{T}$ polymorphism and lung cancer risk in a Chinese population. Anticancer Res 23: 5149-5152, 2003

24. Hosseini M, Houshmand M and Ebrahimi A: MTHFR polymorphisms and breast cancer risk. Arch Med Sci 7: 134-137, 2011.

25. Hubner RA and Houlston RS: MTHFR C677T and colorectal cancer risk: a meta-analysis of 25 populations. Int J Cancer 120: 1027-1035, 2007.

26. Zintzaras E: Association of methylenetetrahydrofolate reductase (MTHFR) polymorphisms with genetic susceptibility to gastric cancer: a meta-analysis. J Hum Genet 51: 618-624, 2006.

27. Issa JP: DNA methylation as a therapeutic target in cancer. Clin Cancer Res 13: 1634-1637, 2007.

28. Kim YI: Folate and carcinogenesis: evidence, mechanisms, and implications. J Nutr Biochem 10: 66-88, 1999.

29. Weisberg IS, Jacques PF, Selhub J, et al: The $1298 \mathrm{~A} \rightarrow \mathrm{C}$ polymorphism in methylenetetrahydrofolate reductase (MTHFR): in vitro expression and association with homocysteine. Atherosclerosis 156: 409-415, 2001.

30. Stern LL, Mason JB, Selhub J and Choi SW: Genomic DNA hypomethylation, a characteristic of most cancers, is present in peripheral leukocytes of individuals who are homozygous for the C677T polymorphism in the methylenetetrahydrofolate reductase gene. Cancer Epidemiol Biomarkers Prev 9: 849-853, 2000.

31. Arslan S, Karadayi S, Yildirim M, Ozdemir O and Akkurt I: The association between methylene-tetrahydrofolate reductase gene polymorphism and lung cancer risk. Mol Biol Rep 38: 991-996, 2011.

32. Hung R, Hashibe M, McKay J, et al: Folate-related genes and the risk of tobacco-related cancers in Central Europe. Carcinogenesis 28: 1334-1340, 2007

33. Shi Q, Zhang Z, Li G, Pillow PC, Hernandez LM, Spitz MR and Wei Q: Sex differences in risk of lung cancer associated with methylene-tetrahydrofolate reductase polymorphisms. Cancer Epidemiol Biomarkers Prev 14: 1477-1484, 2005.

34. Siemianowicz K, Gminski J, Garczorz W, Slabiak N, Goss M, Machalski M and Magiera-Molendowska H: Methylenetetrahydrofolate reductase gene C677T and A1298C polymorphisms in patients with small cell and non-small cell lung cancer. Oncol Rep 10: 1341-1344, 2003

35. Heijmans BT, Boer JM, Suchiman HE, et al: A common variant of the methylenetetrahydrofolate reductase gene (1p36) is associated with an increased risk of cancer. Cancer Res 63 1249-1253, 2003.

36. Shen H, Spitz MR, Wang LE, Hong WK and Wei Q: Polymorphisms of methylene-tetrahydrofolate reductase and risk of lung cancer: a case-control study. Cancer Epidemiol Biomarkers Prev 10: 397-401, 2001.

37. Higgins JPT and Green S (eds): Cochrane Handbook for Systematic Reviews of Interventions Version 5.1.0 (updated March 2011). The Cochrane Collaboration, 2011. Available at www.cochrane-handbook.org.

38. Golledge J and Norman PE: Relationship between two sequence variations in the gene for peroxisome proliferator-activated receptor-gamma and plasma homocysteine concentration. Health in men study. Hum Genet 123: 35-40, 2008

39. Sazci A, Ergul E, Tuncer N, Akpinar G and Kara I: Methylenetetrahydrofolate reductase gene polymorphisms are associated with ischemic and hemorrhagic stroke: Dual effect of MTHFR polymorphisms C677T and A1298C. Brain Res Bull 71: 45-50, 2006. 